\title{
Hyperuricemia is a Risk Factor for One-Year Overall Survival in Elderly Female Patients with Acute Coronary Syndrome
}

\author{
Shi Tai, Xuping Li, Zhaowei Zhu, Liang Tang, Hui Yang, Liyao Fu, Xinqun Hu (D), \\ Zhenfei Fang $\mathbb{D}$, and Shenghua Zhou \\ Department of Cardiology, The Second Xiangya Hospital of Central South University, Changsha, China \\ Correspondence should be addressed to Shenghua Zhou; zhoushenghua@csu.edu.cn
}

Received 7 November 2019; Accepted 29 January 2020; Published 22 February 2020

Academic Editor: Prasanth Puthanveetil

Copyright ( $\odot 2020$ Shi Tai et al. This is an open access article distributed under the Creative Commons Attribution License, which permits unrestricted use, distribution, and reproduction in any medium, provided the original work is properly cited.

\begin{abstract}
Background. Hyperuricemia is a risk factor for cardiovascular diseases, but the impact of hyperuricemia and sex-related disparities is not fully clear in elderly patients with acute coronary syndrome (ACS). Objective. To investigate the association between hyperuricemia and 1-year all-cause mortality in elderly patients with ACS. Methods. This retrospective cohort study included 711 consecutive ACS patients aged $\geq 75$ years, hospitalized in our center between January 2013 and December 2017. Serum uric acid (sUA), in-hospital events, and 1-year follow-up were analyzed. Multivariable logistic regression models were used to explore the risk factors for in-hospital events and 1-year all-cause mortality. Results. sUA levels were higher in males than in females $(381.4 \pm 110.1$ vs. $349.3 \pm 119.1 \mu \mathrm{mol} / 1, P<0.001)$. Prevalence of hypertension $(80.5 \%$ vs. $72.6 \%, P=0.020)$, atrial fibrillation $(16.2 \%$ vs. $9.5 \%, P=0.008)$, and severe heart failure $(61.0 \%$ vs. $44.2 \%, P<0.001)$ were higher in patients with hyperuricemia than in patients with normal sUA. During the 1-year follow-up, 135 patients died (19.0\%); all-cause mortality was higher in patients with hyperuricemia than in patients with normal sUA $(23.1 \%$ vs. $16.7 \%, P=0.039)$. Hyperuricemia is related to in-hospital ventricular tachycardia and 1-year all-cause mortality $(\mathrm{OR}=1.799,95 \% \mathrm{CI} 1.050-3.081, P=0.033 ; \mathrm{OR}=1.512,95 \% \mathrm{CI}$ $1.028-2.225, P=0.036$, respectively). Multivariable regression analysis models showed that hyperuricemia was an independent risk factor of 1-year all-cause mortality in women $(\mathrm{OR}=2.539,95 \% \mathrm{CI} 1.001-6.453, P=0.050)$, but not in $\operatorname{men}(\mathrm{OR}=0.931,95 \%$ CI $0.466-1.858, P=0.839$ ) after adjustment for confounding variables. Conclusions. Hyperuricemia is an independent risk factor for 1-year all-cause mortality in elderly female patients with ACS.
\end{abstract}

\section{Introduction}

The global population aged $>60$ years is increasing continuously now [1-3], the population aged $>60$ years is expected to reach 2 billion by the year of 2050 [2]. Aging is natural and will usually lead to physiological changes that compromise the physical, mental, and functional abilities [4]. Elderly population need to be cared for specially and individually due to increased mobility and comorbidity. Diseases features and risk stratification of elderly acute coronary syndrome (ACS) patients might therefore differ from younger patients. ACS is a critical clinical manifestation referring to a wide spectrum of clinical presentations including ST-segment elevation myocardial infarction (STEMI), non-ST-segment elevation myocardial infarction
(NSTEMI), and unstable angina [5, 6]. As expected, the prevalence of ACS is significantly associated with aging [7], and the outcome of elderly patients with ACS is also worse in this patient group as compared to younger patients with ACS [8]. In fact, almost one out of two patients hospitalized for ACS is $>75$ years of age [9]. Despite the application of various risk stratification schemes in patients with ACS [10-13], risk stratification for elderly ACS patients remains challenging due to the lack of sufficient data and reports.

Substantial evidence suggests that serum uric acid (sUA), as the end product of purine metabolism, is an independent biomarker capable of predicting morbidity and mortality in patients with a variety of cardiovascular diseases (CVD) [14], as well as in healthy young and middle-aged adults [15]. The 2018 expert consensus for the diagnosis and treatment of the 
patient with hyperuricemia [16] highlights the importance of monitoring sUA levels in patients with hyperuricemia and high CV risk $[14,17]$.

Currently available data are less conclusive regarding the role of sUA as an independent risk factor in elderly patients with ACS. Notably, sex-related disparities in this population remain largely unknown [18]. Therefore, this study aimed to investigate the association between hyperuricemia and 1year all-cause mortality in elderly patients with ACS, as well as the sex-related differences.

\section{Methods}

2.1. Study Design and Patients. This was a retrospective cohort study with 711 patients aged $\geq 75$ years and hospitalized in our center for ACS between January 2013 and December 2017. To ensure exhaustive data collection, only patients who were initially admitted to our center were included; those transferred from other centers were not included. The study protocol conformed to the ethical guidelines of the 1975 Declaration of Helsinki as reflected by prior approval from the human research committee of our institution. The need for individual consent was waived by the committee.

2.2. Grouping and Definitions. The study population was divided into two groups according to presence or absence of hyperuricemia. Hyperuricemia was defined as sUA levels of $>416 \mu \mathrm{mol} / \mathrm{l}(>7 \mathrm{mg} / \mathrm{dl})$ in men and $>357 \mu \mathrm{mol} / \mathrm{l}(>6 \mathrm{mg} / \mathrm{dl})$ in women [18].

ACS diagnosis was made according to the standard definitions of the American College of Cardiology [5, 19]. In the present study, severe heart failure referred to Killip class III-IV or New York Heart Association (NYHA) class III-IV. Ventricular tachycardia (VT) was defined as more than 5 beats monitored by 24-hour Holter electrocardiogram (ECG).

2.3. Data Collection. The following information was collected: body weight and height during hospitalization and calculated body mass index (BMI), diabetes, atrial fibrillation (AF), chronic kidney disease, history of chest pain, history of CVD treatment, smoking, laboratory parameters, hospital stay, demographic characteristics, medication, and in-hospital management and events. sUA levels were routinely measured after an overnight fast from peripheral venous blood samples within the first $24-48 \mathrm{~h}$ of hospitalization. The following biochemical parameters were also obtained: hemoglobin, total cholesterol, high-density lipoprotein cholesterol (HDL-C), low-density lipoprotein cholesterol (LDL-C), triglycerides, and glycated hemoglobin A1C (HbA1c).

2.4. Follow-Up. In-hospital outcomes were ascertained by hospital chart review. After discharge, participant follow-up was carried out by means of outpatient visits, telephone calls up to one year.
2.5. Statistical Analysis. SPSS 23.0 (IBM, Armonk, NY, USA) was used for statistical analysis. Continuous variables are presented as means \pm standard deviation, or as median and interquartile range according to their distribution, as determined by the Kolmogorov-Smirnov test; comparisons between groups were performed with the Student's $t$-test or nonparametric tests, as appropriate. Categorical variables are reported as percentage and compared with the chisquare test. Multivariable logistic regression models were used to assess the relationship between risk factors and allcause mortality. To investigate the possible risk factors for mortality in our study, we first investigated the factors of mortality with univariable analyses based on the clinical characteristics. Multivariable logistic regression analysis was applied with the risk factors defined by univariable analyses with $P<0.05$. Estimates of odds ratios (ORs) with their $95 \%$ confidence intervals (CIs) were reported. $P$ values $<0.05$ were considered statistically significant.

\section{Results}

3.1. Characteristics of the Patients. A total of 711 patients were included in the analysis (438 men and 273 women). The sUA concentrations ranged from 107.4 to $942.1 \mu \mathrm{mol} / \mathrm{l}$ (mean \pm SD, $369.1 \pm 114.6 \mu \mathrm{mol} / \mathrm{l})$. sUA levels were significantly higher in males than in females $(381.4 \pm 110.1$ vs. $349.2 \pm 119.1 \mu \mathrm{mol} / \mathrm{l}$; $P<0.001)$. There were $182(25.8 \%)$ STEMI and $529(74.2 \%)$ NSTEMI patients; prevalence of hyperuricemia was similar between the two groups $(34.1 \%$ vs. $35.7 \%)$.

3.2. Characteristics of the Patients according to $s U A$. There were $251(35.3 \%)$ patients in the hyperuricemia group and $460(64.7 \%)$ patients in the normal sUA group. The characteristics of each group are listed in Table 1. Body mass index (BMI), prevalence of hypertension, previous chest pain, previous percutaneous coronary intervention (PCI), and stroke were similar between the two groups. The incidence of hypertension ( $80.5 \%$ vs. $72.6 \%, P=0.020)$, AF (16.2\% vs. 9.5\%, $P=0.008)$, and severe heart function $(61.0 \%$ vs. $44.2 \%, P<0.001)$ were significantly higher in patients with hyperuricemia than in patients with normal sUA.

The clinical features at admission are shown in Table 2. Hemoglobin $(119.3 \pm 19.2$ vs. $113.9 \pm 21.6 \mathrm{~g} / \mathrm{l}, P=0.001)$ and HDL-cholesterol $(1.1 \pm 0.3$ vs. $1.0 \pm 0.3 \mathrm{mmol} / \mathrm{L}, P=0.001)$ were significantly lower, whereas creatinine levels $(138.0 \pm 106.8$ vs. $96.6 \pm 68.1 \mu \mathrm{mol} / 1, P<0.001)$ and NTproBNP (887.3 [290.0-2656.2] vs. 1704.0 [536.6-5077.2], $P<0.001)$ were significantly higher in the hyperuricemia group than in normal sUA group.

In-hospital management information is shown in Supplemental Table 1. Medication was similar between the two groups, except that aspirin $(88.6 \%$ vs. $78.5 \%, P<0.001)$ and diuretics ( $44.1 \%$ vs. $55.9 \%, P=0.003$ ) were used more often in the hyperuricemia group than in the non-hyperuricemia group.

3.3. In-Hospital Events and 1-Year Outcomes. In-hospital events are shown in Table 3 . There were no significant 
TABLE 1: Baseline characteristics of patients according to sUA levels.

\begin{tabular}{|c|c|c|c|}
\hline Parameters & sUA normal $(n=460)$ & Hyperuricemia $(n=251)$ & $P$ \\
\hline \multicolumn{4}{|l|}{ Demographics and medical history } \\
\hline Age, years (median, IQR) & $78(76-80)$ & $78(76-81)$ & 0.308 \\
\hline Female sex, $n(\%)$ & $164(35.7)$ & $109(43.4)$ & 0.042 \\
\hline Body mass index (median, IQR) & $22.8(20.8-25.0)$ & $23.4(20.8-26.3)$ & 0.101 \\
\hline Diabetes mellitus, $n(\%)$ & $138(30.0)$ & $74(29.5)$ & 0.885 \\
\hline Hypertension, $n(\%)$ & $334(72.6)$ & $202(80.5)$ & 0.020 \\
\hline Atrial fibrillation, $n(\%)$ & $43(9.5)$ & $40(16.2)$ & 0.008 \\
\hline $\mathrm{CKD} \geq 3$ & $17(3.7)$ & $22(8.8)$ & 0.005 \\
\hline Stroke, $n(\%)$ & $70(15.5)$ & $33(13.5)$ & 0.481 \\
\hline Previous chest pain, $n(\%)$ & $299(65.9)$ & $168(68.3)$ & 0.514 \\
\hline Previous PCI, $n(\%)$ & $82(18.1)$ & $32(13.0)$ & 0.082 \\
\hline Previous CABG, $n(\%)$ & $9(2.0)$ & $4(1.6)$ & 0.739 \\
\hline Current smoking, $n(\%)$ & $171(38.5)$ & $82(34.2)$ & 0.261 \\
\hline \multicolumn{4}{|l|}{ Examination findings } \\
\hline SBP, mmHg (median, IQR) & $134(117-152)$ & $132(118-152)$ & 0.520 \\
\hline $\mathrm{DBP}, \mathrm{mmHg}$ (median, IQR) & $72(65-81)$ & $71(64-80)$ & 0.184 \\
\hline Heart rate, beats/min (median, IQR) & $75(67-84)$ & $76(68-86)$ & 0.158 \\
\hline \multicolumn{4}{|l|}{ Clinical presentation } \\
\hline Severe heart failure, $n(\%)$ & $203(44.2)$ & $153(61.0)$ & $<0.001$ \\
\hline STEMI, $n(\%)$ & $120(26.1)$ & $62(24.7)$ & 0.686 \\
\hline NSTE-ACS, $n(\%)$ & $340(73.9)$ & $189(75.3)$ & 0.686 \\
\hline
\end{tabular}

sUA: serum uric acid; IQR: interquartile range; CKD: chronic kidney disease; PCI: percutaneous coronary intervention; CABG: coronary artery bypass graft; SBP: systolic blood pressure; DBP: diastolic blood pressure; STEMI: ST-elevated myocardial infarction; NSTE-ACS: non-ST-elevation acute coronary syndrome.

TABle 2: Clinical data on admission of patients according to sUA levels.

\begin{tabular}{|c|c|c|c|}
\hline Clinical data & sUA normal $(n=460)$ & Hyperuricemia $(n=251)$ & $P$ \\
\hline WBC, $10^{9} / \mathrm{L}($ mean $\pm \mathrm{SD})$ & $7.2 \pm 3.2$ & $7.5 \pm 3.2$ & 0.252 \\
\hline Hemoglobin, g/L (mean \pm SD) & $119.3 \pm 19.2$ & $113.9 \pm 21.6$ & 0.001 \\
\hline Platelets, $10^{9} / \mathrm{L}($ mean $\pm \mathrm{SD})$ & $188.7 \pm 64.2$ & $179.0 \pm 64.0$ & 0.058 \\
\hline Fasting glucose, mmol/l (median, IQR) & $5.9(4.8-7.6)$ & $5.6(4.8-6.7)$ & 0.284 \\
\hline HbA1C, \% (median, IQR) & $6.3(5.7-6.9)$ & $6.1(5.6-6.7)$ & 0.313 \\
\hline ALT, U/l (median, IQR) & $18.8(12.8-31.8)$ & $18.8(12.7-33.3)$ & 0.948 \\
\hline AST, U/l (median, IQR) & $22.9(17.3-53.2)$ & $22.3(18.1-37.0)$ & 0.955 \\
\hline Albumin, $g / 1($ mean $\pm S D)$ & $35.1 \pm 4.0$ & $35.0 \pm 4.6$ & 0.688 \\
\hline Triglycerides, mmol/l $($ mean $\pm S D)$ & $1.6 \pm 4.1$ & $1.6 \pm 1.1$ & 0.901 \\
\hline Total cholesterol, mmol/l (mean $\pm \mathrm{SD})$ & $3.9 \pm 0.9$ & $3.9 \pm 1.1$ & 0.365 \\
\hline HDL-cholesterol, mmol/l (mean \pm SD) & $1.1 \pm 0.3$ & $1.0 \pm 0.3$ & 0.019 \\
\hline LDL-cholesterol, mmol/l $($ mean $\pm \mathrm{SD})$ & $2.3 \pm 0.8$ & $2.33 \pm 0.9$ & 0.211 \\
\hline Creatinine, $\mu \mathrm{mol} / \mathrm{l}($ mean $\pm \mathrm{SD})$ & $96.6 \pm 68.1$ & $138.0 \pm 106.8$ & $<0.001$ \\
\hline $\mathrm{PT}, s($ mean $\pm \mathrm{SD})$ & $13.3 \pm 2.5$ & $14.1 \pm 4.7$ & 0.117 \\
\hline $\mathrm{APTT}, s($ mean $\pm \mathrm{SD})$ & $42.3 \pm 21.9$ & $36.8 \pm 11.9$ & 0.101 \\
\hline $\mathrm{CK}, \mathrm{U} / \mathrm{l}($ mean $\pm \mathrm{SD})$ & $350.8 \pm 714.9$ & $294.6 \pm 524.1$ & 0.561 \\
\hline $\mathrm{CK}-\mathrm{Mb}, \mathrm{U} / \mathrm{l}($ mean $\pm \mathrm{SD})$ & $42.0 \pm 102.8$ & $38.4 \pm 104.7$ & 0.681 \\
\hline TnT, pg/ml (median, IQR) & $20.1(11.3-1268.5)$ & $46.7(12.2-1418.3)$ & 0.365 \\
\hline hs-CRP, mg/l (median, IQR) & $4.2(1.2-23.2)$ & $7.0(1.8-20.5)$ & 0.173 \\
\hline NT-proBNP, pg/ml (median, IQR) & $887.3(290.0-2656.2)$ & $1704.0(536.6-5077.2)$ & $<0.001$ \\
\hline $\mathrm{EF}, \%($ mean $\pm \mathrm{SD})$ & $54 \pm 10.1$ & $52 \pm 11.8$ & 0.072 \\
\hline
\end{tabular}

sUA: serum uric acid; WBC: white blood cells; HbAlc: glycated hemoglobin; ALT: alanine aminotransferase; AST: aspartate aminotransferase; HDL: highdensity lipoprotein; LDL: low-density lipoprotein; PT: prothrombin time; APTT: activated prothrombin time; CK: creatine kinase; CK-Mb: creatine kinase $\mathrm{Mb}$ chain; TnT: troponin T; hs-CRP: high-sensitivity C-reactive protein; NR-proBNP: N-terminal prohormone of brain natriuretic peptide; EF: ejection fraction.

differences in severe heart failure $(15.0 \%$ vs. $20.8 \%$, $P=0.053)$, bleeding $(7.5 \%$ vs. $11.1 \%, P=0.113)$, stroke $(1.3 \%$ vs. $0.4 \%, P=0.244)$, and death $(3.4 \%$ vs. $5.8 \%$, $P=0.334)$ between the hyperuricemia and non-hyperuricemia groups, whereas the frequency of VT (11.1\% vs. $6.8 \%$,
$P=0.040$ ) was significantly higher in the hyperuricemia group than in the non-hyperuricemia group. Hyperuricemia is related to increased risk of in-hospital VT (unadjusted $\mathrm{OR}=1.799$, 95\% CI 1.050-3.081, $P=0.033$ ). Besides, hyperuricemia is an independent factor associated with the 
TABLE 3: In-hospital events and follow-up for patients with normal sUA or hyperuricemia.

\begin{tabular}{lccc}
\hline & sUA normal $(n=460)$ & Hyperuricemia $(n=251)$ & \\
\hline In-hospital events & & & \\
Heart failure, $n(\%)$ & $68(15.0)$ & $51(20.8)$ & $27(11.1)$ \\
Bleeding, $n$ (\%) & $34(7.5)$ & $28(11.3)$ & 0.053 \\
VT, $n$ (\%) & $31(6.8)$ & $1(0.4)$ & 0.113 \\
Stroke, $n$ (\%) & $6(1.3)$ & $5(4.8)$ & 0.040 \\
Death, $n$ (\%) & $7(3.7)$ & $5(2.3)$ & 0.244 \\
\hline One-year follow-up & & $97(42.7)$ & 0.642 \\
Revascularization & $6(1.5)$ & $7(3.3)$ & 0.432 \\
Readmission, $n(\%)$ & $158(36.7)$ & $154(67.2)$ & 0.129 \\
Stroke, $n(\%)$ & $24(5.9)$ & $58(23.1)$ & 0.159 \\
Bleeding, $n(\%)$ & $294(68.2)$ & & 0.801 \\
Death, $n(\%)$ & $77(16.7)$ & & 0.039 \\
\hline
\end{tabular}

sUA: serum uric acid; VT: ventricular tachycardia.

appearance of AF $(\mathrm{OR}=2.118,95 \%$ CI 1.126-3.985, $P=0.020)$ after adjustment for confounding variables including age, sex, BMI, current smoking, hypertension, diabetes mellitus, PCI or CABG history, $\mathrm{CKD} \geq 3$, stroke, ACS type, and severe heart failure. A total of 135 patients (19.0\%) died during 1-year follow-up, and the all-cause mortality rate was significantly higher in patients with hyperuricemia than in patients with normal sUA $(23.1 \%$ vs. $16.7 \%$, $P=0.039$ ). Patients with hyperuricemia had significantly higher unadjusted hazard of death (unadjusted $\mathrm{OR}=1.512$, 95\% CI 1.028-2.225, $P=0.036$ ) than patients with normal sUA group. Rehospitalization, revascularization, stroke, and bleeding after discharge were similar between the two groups.

3.4. Characteristics of the Patients according to Sex. Sex-related disparities are shown in Table 4. The prevalence of hyperuricemia was significantly higher in females than in males (39.9\% vs. $32.4 \%, P=0.042$ ). Female patients had higher frequencies of diabetes mellitus $(35.2 \%$ vs. $26.5 \%, P=0.014)$ and NSTE-ACS (79.5\% vs. $72.1 \%, P=0.028)$; lower frequencies of STEMI (20.5\% vs. $26.9 \%, P=0.028)$ and smoking (5.4\% vs. $56.5 \%, P<0.001)$; higher frequency of severe heart failure on admission (56.8\% vs. $46.0 \%, P=0.005$ ); higher total cholesterol $(4.1 \pm 1.0$ vs. $3.8 \pm 0.9 \mathrm{mmol} / \mathrm{l}, P<0.001)$, HDL-C $(1.1 \pm 0.3$ vs. $1.0 \pm 0.3 \mathrm{mmol} / \mathrm{L}, P=0.002)$, LDL-C ( $2.4 \pm 0.9$ vs. $2.2 \pm 0.8 \mathrm{mmol} / \mathrm{L}, \quad P=0.002)$, platelets $(202.9 \pm 64.9$ vs. $174.3 \pm 61.4 \times 10^{9} / \mathrm{L}, P<0.001$ ), and HbA1c (median, $6.7 \%$ vs. $6.1 \%, P=0.005)$; and lower hemoglobin (110.4 \pm 18.3 vs. $121.8 \pm 20.2 \mathrm{~g} / \mathrm{L}, P<0.001$ ), alanine aminotransferase (ALT) (median, 17.5 vs. $19.9 \mathrm{U} / \mathrm{L}, P=0.012)$, creatinine $(89.9 \pm 54.1$ vs. $124.4 \pm 98.6 \mu \mathrm{mol} / \mathrm{L}, P<0.001)$, and sUA $(349.2 \pm 119.1$ vs. $381.4 \pm 110.1 \mu \mathrm{mol} / \mathrm{L}, P<0.001)$ than male patients. The inhospital mortality rate was similar $(3.5 \%$ vs. $4.4 \%, P=0.693)$, but male patients had higher 1-year all-cause mortality $(21.7 \%$ vs. $14.7 \%, P=0.020)$. Although patients with hyperuricemia had more severe heart failure on admission $(66.1 \%$ vs. $50.6 \%$, $P=0.012 ; 57.0 \%$ vs. $40.7 \%, P=0.012$, respectively) and higher creatine $\quad(111.1 \pm 69.2$ vs. $\quad 76.0 \pm 35.3 \mu \mathrm{mol} / \mathrm{L}, \quad P<0.001$; $158.3 \pm 124.4$ vs. $108.0 \pm 78.5 \mu \mathrm{mol} / \mathrm{L}, P<0.001$, respectively) and sUA $(462.0 \pm 101.3$ vs. $274.4 \pm 52.0 \mu \mathrm{mol} / \mathrm{L}, P<0.001$; $504.1 \pm 84.3$ vs. $322.6 \pm 61.9 \mu \mathrm{mol} / \mathrm{L}, P<0.001$, respectively) than those without hyperuricemia in female and male population, respectively, female patients with hyperuricemia had a higher frequency of $\mathrm{CKD} \geq 3$ (9.2\% vs. $1.8 \%, P=0.005)$, lower EF value ( $51.6 \pm 11.7$ vs. $55 \pm 9.2 \%, P=0.009)$, and higher 1 year all-cause mortality $(22.0 \%$ vs. $9.8 \%, P=0.005)$ than those without hyperuricemia, but mortality was similar in male patients with or without hyperuricemia.

3.5. Multivariable Analyses. We examined the independent determinants of 1-year all-cause mortality. As shown in Supplemental Table 2, female patients with hyperuricemia had significantly higher unadjusted odds of death (unadjusted $\mathrm{OR}=2.612,95 \%$ CI $1.315-5.189, P=0.006$ ) than female patients with normal sUA. Similar results were observed $(\mathrm{OR}=2.438,95 \% \mathrm{CI} 1.215-4.894, P=0.012)$ after adjusting for age (Model 1). The same associations were observed in model $2(\mathrm{OR}=2.643$, 95\% CI 1.092-6.400, $P=0.031$ ) after adjustment for age, BMI, current smoking, hypertension, and diabetes mellitus and in model 3 $(\mathrm{OR}=2.539,95 \%$ CI 1.001-6.453, $P=0.050)$ after adjustment for age, BMI, current smoking, PCI or CABG history, hypertension, diabetes mellitus, $\mathrm{CKD} \geq 3$, and stroke. The multivariable analysis showed that hyperuricemia was not an independent risk factor of 1-year all-cause mortality for male patients and for the total cohort $(\mathrm{OR}=0.931,95 \% \mathrm{CI}$ $0.466-1.858, P=0.839 ; \mathrm{OR}=1.326,95 \%$ CI $0.773-2.273$, $P=0.305$, respectively).

\section{Discussion}

Hyperuricemia is a risk factor for CVDs, but the impact of hyperuricemia and sex-related disparities are not fully clear in elderly patients with ACS. Therefore, this study aimed to investigate the association between hyperuricemia and outcome in elderly patients with ACS. The results suggest that patients with hyperuricemia have higher in-hospital events and 1-year all-cause mortality compared with those without hyperuricemia. Importantly, hyperuricemia is an independent risk factor for one-year all-cause mortality after discharge in elderly female patients with ACS, but not in elderly male patients with ACS. 


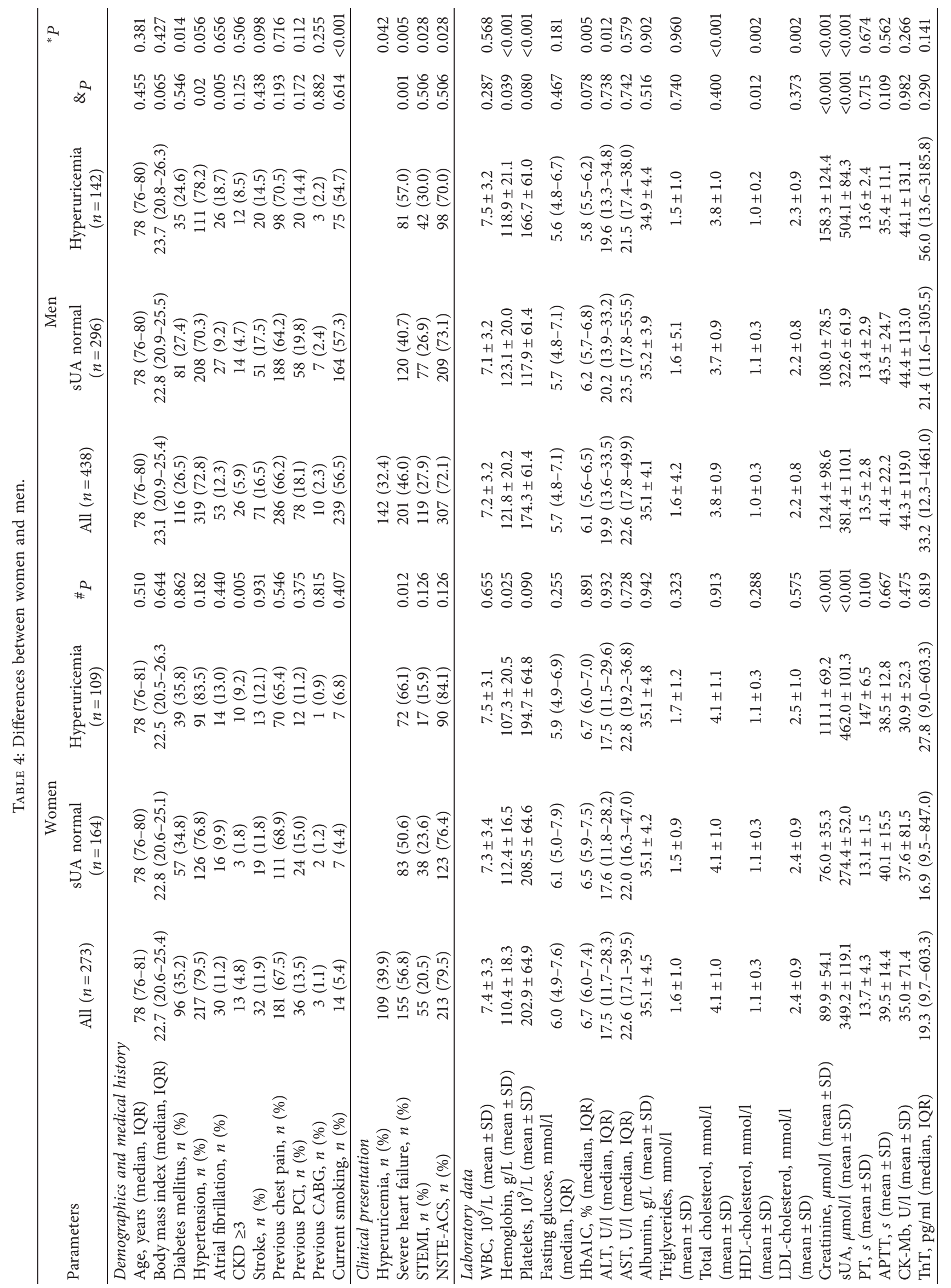




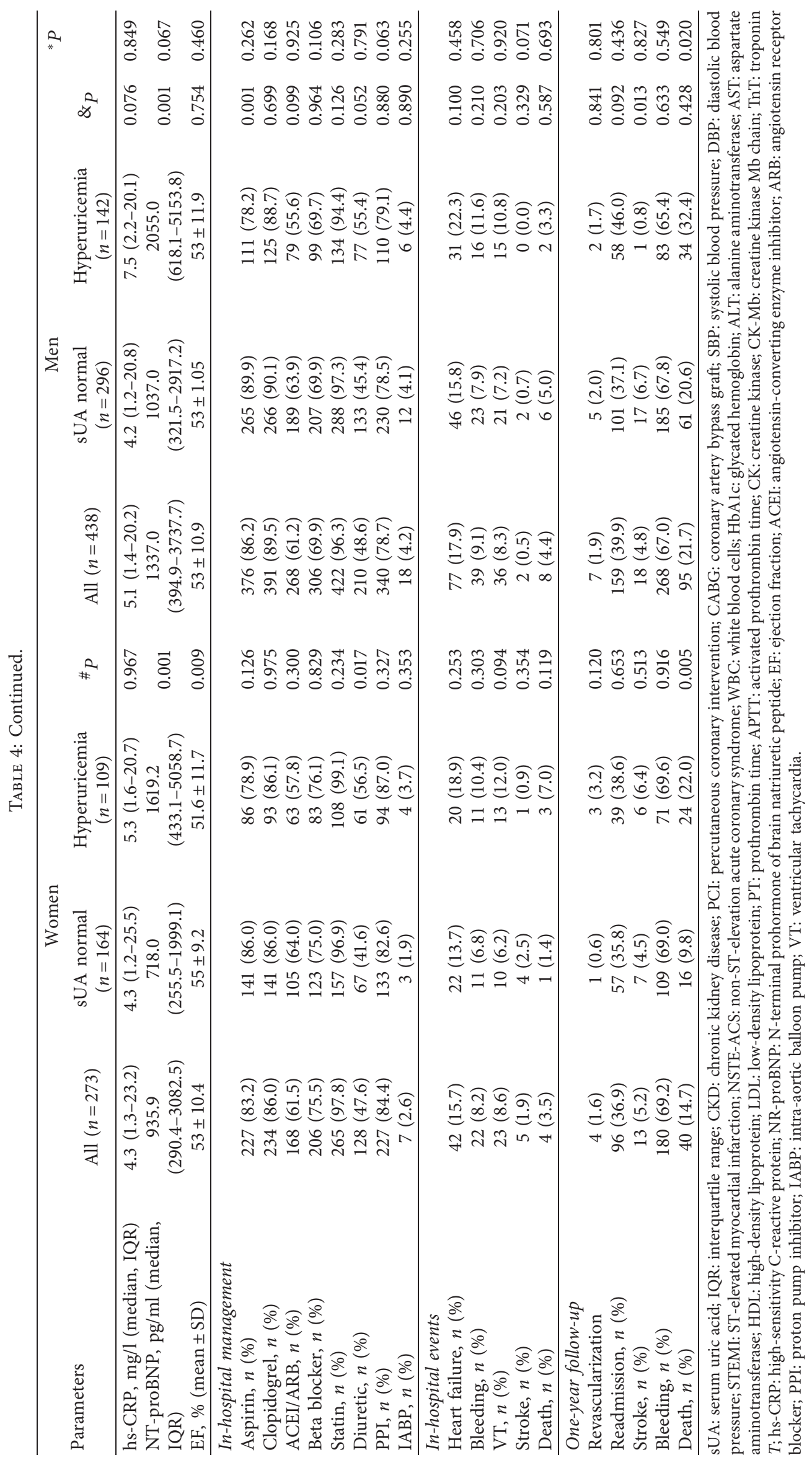


CVDs are the leading causes of death worldwide [20]. The high mortality in elderly patients with ACS $[1,8]$ justifies the cumulative risk factors, which might result in poor outcome in this patient population. The present study showed a rate of $19.0 \%$ for 1-year all-cause mortality in elderly patients with ACS, which is consistent with a previous report [21]. A large body of evidence indicates that hyperuricemia is an emerging CVD risk factor [22, 23], besides traditional risk factors for CVD. The results of the present study showed that hyperuricemia is associated with the female sex, arterial hypertension, AF, lower HDL-cholesterol, elevated serum creatinine, and in-hospital cardiac complications. It is likely that the higher in-hospital event and one-year all-cause mortality rates after discharge are the consequences of the more frequent clustering of these risk factors, and hyperuricemia might thus be viewed as a surrogate risk factor in elderly ACS patients. The multivariable analyses showed that hyperuricemia is an independent determinant of one-year all-cause mortality in female ACS patients, but not in males or in the whole cohort after adjustment for age, BMI, current smoking, PCI or CABG history, hypertension, diabetes mellitus, $C K D \geq 3$, and stroke, as supported by previous studies [24-27]. A previous report from a large Chinese cohort showed that the prevalence of hyperuricemia was $42.6 \%$ in men and $23.4 \%$ in women [18]; we found that the prevalence of hyperuricemia is even higher in elderly female patients with ACS than in their male counterparts. This pattern confirms the presence of sex-specific differences in elderly patients with ACS in terms of hyperuricemia. This discrepancy could be attributed to menopausal women having higher sUA levels than premenopausal women [28]. Women with ACS, especially at age $\geq 75$, have been described as being sicker than their male counterparts [29]. Similarly, our data also indicate that elderly female patients had more risk factors for CVD, including diabetes mellitus, hypertension, and dyslipidemia. Nevertheless, male patients had worse 1-year outcomes compared with those of women. The possible reason might be that male elderly ACS patients have other special risk factors, such as higher frequencies of STEMI, current smoking, and worse renal function, which may influence the 1 -year all-cause mortality. It was shown that the prevalence of hyperuricemia in general population was $21.4 \%$ (21.2\% for men and $21.6 \%$ for women) according to the United States National Health and Nutrition Examination Survey (NHANES) study [30]. It was also shown that the prevalence of hyperuricemia increased with aging in male and female (14.2\% at age $<30,20.1 \%$ at age $31-40,22.9 \%$ at age $41-50$, $22.3 \%$ at age $51-60,23.3 \%$ at age $61-70$, and $24.1 \%$ at age $>70$ in men; $2.8 \%$ at age $<30,3.5 \%$ at age $31-40,6.6 \%$ at age $41-50,14.7 \%$ at age $51-60,16.8 \%$ at age $61-70$, and $23.4 \%$ at age $>70$ in women) $[31,32]$. Our study showed that hyperuricemia served as an independent risk factor in elderly female patients with ACS, implicating the importance of monitoring sUA levels in elderly female ACS patients. Future studies are warranted to verify the prognostic importance of hyperuricemia in ACS patients with various ages and sexes. Moreover, it is of importance to observe that more intensive care, including targeting blood uric acid levels on outcome among elderly female ACS patients, is required.

NHANES III trial showed an increased risk of all-cause death and cardiac death in patients with increased sUA levels, and the association remained significant even after adjustment for different factors including demography and comorbidities [33]. On the other hand, our study showed that hyperuricemia was not associated with in-hospital death and 1-year death in elderly ACS patients. The reason for these discrepancies remains elusive. Although hyperuricemia is a risk factor for 1-year all-cause mortality in the present study, we failed to confirm the independent association between hyperuricemia and 1-year all-cause mortality after adjustment for age and other risk factors, suggesting that, in elderly ACS patients, other comorbidities might serve as more important causes of death than hyperuricemia. Notably, few studies have clarified the relationship between hyperuricemia and sex-specific differences in the outcomes of elderly patients with ACS. Our data indicated that female patients with hyperuricemia have higher frequency of $C K D \geq 3$, lower EF value, and higher 1year all-cause mortality than those without hyperuricemia, but this is not observed in males. Importantly, hyperuricemia was associated with an increased risk of 1-year allcause mortality in elderly women independently of traditional CVD risk factors, but this similar association was not observed in men and in the total population. These findings suggest that hyperuricemia is associated with 1-year allcause mortality more strongly in women than in men with ACS. A previous study reported that changes in sUA levels in menopausal women is associated with sex hormones [34], thereby sex hormones may partly explain the different risk of hyperuricemia in males and females. Given that the mechanisms behind these sex-specific differences in ACS remain unclear, the representation of women in clinical CVD trials needs to be increased.

The mortality rate was higher in men than in women, which is consistent with the general CVD mortality pattern observed between men and women [3, 20,29]. Our findings indicate that, despite lower 1-year mortality among elderly female ACS patients as compared to elderly male ACS patients, hyperuricemia is still considered as an independent risk factor of one-year mortality in elderly female patients, and future studies are warranted to see if more intensive care including therapy targeting hyperuricemia could further reduce the one-year mortality for elderly female ACS patients. Besides, lifestyle habits may also play a role in this difference in mortality. Indeed, in the present study, men had a higher frequency of current smoking than women ( $57 \%$ vs. $5 \%$ ), which is consistent with the pattern observed in the Chinese population [35]. Smoking is a well-known risk factor for all-cause and CVD death [35]. In addition, men had a higher frequency of STEMI, while women had a higher frequency of severe heart failure. Although the difference was small (but significant), women had higher levels of total, LDL-C and HDL-C than men, but the higher levels of HDL-C probably counteracted the higher LDL-C levels, but this will have to be verified more specifically. Recent 
evidence suggests that diabetes has a more significant impact on CVD mortality in women than in men [36], but in the present study, mortality in women was lower than in men despite the frequency of diabetes being higher in women; this will have to be investigated.

Lately, a considerable number of reports associating elevated sUA with cardiac rhythm disorders (such as AF) have been published $[37,38]$. In the present study, the result indicated that hyperuricemia was an independent factor associated with the frequency of $\mathrm{AF}(\mathrm{OR}=2.118,95 \% \mathrm{CI}$ 1.126-3.985, $P=0.020$ ) after adjustment for confounding variables. Gang Huang et al. [39] investigated hyperuricemia and the risk of AF in the very elderly community and found that participants with hyperuricemia had a higher risk of suffering from AF in very elderly Chinese. Leonardo Tamariz et al. [40] identified 1,085 cases of incident AF. In Cox proportional hazards models adjusted for age, gender, race, center, education, and traditional risk factors at baseline, the hazard ratio of $\mathrm{AF}$ associated with a SD increment in sUA was 1.16 (95\% confidence interval 1.06 to 1.26 ). These results indicated that elevated sUA is associated with a greater risk of AF. It is generally accepted that both inflammatory-dependent and -independent mechanisms might play a role in rhythm disorders [41, 42]. Elevated concentrations of sUA via xanthine oxidase system activation are related to the generation of oxidative stress and inflammatory mediators [16]. Oxidative stress promotes calcium overload and decreases sodium channels, resulting in electrical remodeling [43]. Proinflammatory molecules induced by oxidative injury are also increased [44]. At the same time, structural remodeling may be mediated by fibroblasts [43]. Subsequently, electrical and structural remodeling may lead to AF [45]. Regarding the association between increased sUA levels and ventricular arrhythmias, the evidence is sparse. The present study strongly suggests that patients with hyperuricemia had higher odds than those with normal sUA according to the univariable logistic regression analyses, and this association was lost after adjusting for other confounders. A small observational study of individuals with ECG evidence of hypertrophy divided into two groups based on whether VT was detected or not on a 24-h ECG recording, demonstrated that sUA was an independent factor associated with the appearance of VT [46], but one should keep in mind that arrhythmia has a complex pathophysiologic background. The limited available data only explain the possible relationship and conceptual framework, and additional research may be needed to clarify the underlying mechanisms.

4.1. Limitations. This study had some limitations. First, this is a single center experience and the number of patients was relatively small when considering the high incidence of ACS. Therefore, we set a strict standard for the selection of potential variables $(P<0.05)$ for the multivariable logistic analysis, and we might have missed some factors. Besides, the observational study design allows determining only associations and not causality; the mechanisms behind the results obtained require further investigation. Finally,
STEMI and NSTE-ACS patients are quite heterogeneous regarding symptoms and outcomes, yet these patients were not analyzed separately in this study.

\section{Conclusion}

Hyperuricemia is associated with in-hospital events and 1year all-cause mortality, and it is an independent risk factor for 1-year all-cause mortality after discharge in elderly female patients with ACS. Monitoring sUA levels would be helpful to clinicians treating these elderly patients suffering from hyperuricemia and ACS.

\section{Data Availability}

The data used to support the findings of this study are included within the supplementary information file.

\section{Additional Points}

Elderly ACS patients with hyperuricemia have higher inhospital events and 1-year all-cause mortality compared with those without hyperuricemia. Hyperuricemia is an independent risk factor for one-year all-cause mortality after discharge in elderly female patients with ACS, but not in elderly male patients with ACS.

\section{Conflicts of Interest}

The authors declare that they have no conflicts of interest.

\section{Authors' Contributions}

Shi Tai, Xuping Li, and Zhaowei Zhu contributed equally. All authors collaborated to carry out the work presented here. Shenghua Zhou defined the study theme and methods. Shi Tai, Xuping Li, and Zhaowei Zhu collected clinical data, analyzed the data, interpreted the results, and wrote the paper. Liang Tang, Hui Yang, Xinqun Hu, and Zhenfei Fang are the attending doctors responsible for the treatment of those patients. Liyao $\mathrm{Fu}$ is the research assistant responsible for the laboratory test. All authors have contributed to, seen, and approved the manuscript.

\section{Acknowledgments}

This research was supported by the Natural Science Foundation of China (81670269 to Shenghua Zhou and 81801394 to Shi Tai).

\section{Supplementary Materials}

The supplementary materials contain two tables. In-hospital management information is shown in Supplemental Table 1, and the independent determinants of 1-year all-cause mortality are shown in Supplemental Table 2. And the supplementary Excel file is the datasheet used for the statistical analysis. (Supplementary Materials) 


\section{References}

[1] L. Faroux, S. Tassan-Mangina, B. Herce, P. Nazeyrollas, K. Bauley, and D. Metz, "Acute coronary syndromes in the very elderly: short term prognostic performance of the SYNTAX score," International Journal of Cardiology, vol. 243, pp. 497-501, 2017.

[2] United Nations Department of Economic and Social Affairs, World Population Ageing, 1950-2050, United Nations Department of Economic and Social Affairs, New York, NY, USA, 2002.

[3] W. Lutz, W. Sanderson, and S. Scherbov, "The coming acceleration of global population ageing," Nature, vol. 451, no. 7179, pp. 716-719, 2008.

[4] J. J. Yue, The Comprehensive Treatment of the Aging Spine Minimally Invasive and Advanced Techniques, Saunders/ Elsevier, Philadelphia, PA, USA, 1st edition, 2011.

[5] E. A. Amsterdam, N. K. Wenger, R. G. Brindis et al., "2014 AHA/ACC guideline for the management of patients with non-ST-elevation acute coronary syndromes," Journal of the American College of Cardiology, vol. 64, no. 24, pp. e139-e228, 2014.

[6] E. Braunwald, "Unstable angina and non-ST elevation myocardial infarction," American Journal of Respiratory and Critical Care Medicine, vol. 185, no. 9, pp. 924-932, 2012.

[7] M. Magnoni, M. Berteotti, F. Ceriotti et al., "Serum uric acid on admission predicts in-hospital mortality in patients with acute coronary syndrome," International Journal of Cardiology, vol. 240, pp. 25-29, 2017.

[8] X. Dai, J. Busby-Whitehead, and K. P. Alexander, "Acute coronary syndrome in the older adults," Journal of Geriatric Cardiology, vol. 13, no. 13, pp. 101-108, 2016.

[9] D. D. McManus, J. Gore, J. Yarzebski, F. Spencer, D. Lessard, and R. J. Goldberg, "Recent trends in the incidence, treatment, and outcomes of patients with STEMI and NSTEMI," The American Journal of Medicine, vol. 124, no. 1, pp. 40-47, 2011.

[10] A. C. Fanaroff, A. Y. Chen, L. E. Thomas et al., "Risk score to predict need for intensive care in initially hemodynamically stable Adults with non-ST-segment-elevation myocardial infarction," Journal of the American Heart Association, vol. 7, no. 11, 2018.

[11] U. N. Khot, G. Jia, D. J. Moliterno et al., "Prognostic importance of physical examination for heart failure in non-STelevation acute coronary syndromes," JAMA, vol. 290, no. 16, pp. 2174-2181, 2003.

[12] P. Vranckx, H. D. White, Z. Huang et al., "Validation of BARC bleeding criteria in patients with acute coronary syndromes," Journal of the American College of Cardiology, vol. 67, no. 18, pp. 2135-2144, 2016.

[13] D. Poçi, M. Hartford, T. Karlsson, J. Herlitz, N. Edvardsson, and K. Caidahl, "Role of the CHADS 2 score in acute coronary syndromes," Chest, vol. 141, no. 6, pp. 1431-1440, 2012.

[14] C. Borghi, E. A. Rosei, T. Bardin et al., "Serum uric acid and the risk of cardiovascular and renal disease," Journal of $\mathrm{Hy}$ pertension, vol. 33, no. 9, pp. 1729-1741, 2015.

[15] T. D. S. Ferreira, J. F. R. Fernandes, L. D. S. Araújo et al., "Serum uric acid levels are associated with cardiometabolic risk factors in healthy young and middle-aged adults," Arquivos Brasileiros de Cardiologia, vol. 111, no. 111, pp. 833-840, 2018.

[16] C. Borghi, A. Tykarski, K. Widecka et al., "Expert consensus for the diagnosis and treatment of patient with hyperuricemia and high cardiovascular risk," Cardiology Journal, vol. 25, no. 5, pp. 545-563, 2018.
[17] G. Ruoff and N. L. Edwards, "Overview of serum uric acid treatment targets in gout: why less than $6 \mathrm{mg} / \mathrm{dL}$ ?," Postgraduate Medicine, vol. 128, no. 7, pp. 706-715, 2016.

[18] S.-Y. Chuang, J.-H. Chen, W.-T. Yeh, C.-C. Wu, and W.-H. Pan, "Hyperuricemia and increased risk of ischemic heart disease in a large Chinese cohort," International Journal of Cardiology, vol. 154, no. 3, pp. 316-321, 2012.

[19] P. T. O'Gara, F. G. Kushner, D. D. Ascheim et al., “2013 ACCF/AHA guideline for the management of ST-elevation myocardial infarction: a report of the American College of Cardiology Foundation/American Heart Association Task Force on Practice Guidelines," Circulation, vol. 127, no. 4, pp. e362-425, 2013.

[20] M. Heron, “Deaths: leading causes for 2007," National Vital Statistics Reports, vol. 59, no. 8, pp. 1-95, 2011.

[21] Z. Zhaowei, P. Xiaofan, X. Hebin et al., "Comparison of oneyear survival after acute coronary syndrome in patients $\geq 75$ years of age with versus without living with spouse," The American Journal of Cardiology, vol. 123, no. 1, pp. 1-6, 2019.

[22] M. Bove, A. F. G. Cicero, M. Veronesi, and C. Borghi, "An evidence-based review on urate-lowering treatments: implications for optimal treatment of chronic hyperuricemia," Vascular Health and Risk Management, vol. 13, pp. 23-28, 2017.

[23] C. Borghi and G. Desideri, "Urate-Lowering drugs and prevention of cardiovascular disease," Hypertension, vol. 67, no. 3, pp. 496-498, 2016.

[24] B. F. Culleton, M. G. Larson, W. B. Kannel, and D. Levy, "Serum uric acid and risk for cardiovascular disease and death: the Framingham Heart Study," Annals of Internal Medicine, vol. 131, no. 1, pp. 7-13, 1999.

[25] J. T. Moriarity, A. R. Folsom, C. Iribarren, F. J. Nieto, and W. D. Rosamond, "Serum uric acid and risk of coronary heart disease," Annals of Epidemiology, vol. 10, no. 3, pp. 136-143, 2000.

[26] S. G. Wannamethee, A. G. Shaper, and P. H. Whincup, "Serum urate and the risk of major coronary heart disease events," Heart, vol. 78, no. 2, pp. 147-153, 1997.

[27] F. N. Brand, D. L. McGee, W. B. Kannel, J. Stokes III, and W. P. Castelli, "Hyperuricemia as a risk factor of coronary heart disease: the Framingham Study," American Journal of Epidemiology, vol. 121, no. 1, pp. 11-18, 1985.

[28] D. I. Feig, D.-H. Kang, and R. J. Johnson, "Uric acid and cardiovascular risk," New England Journal of Medicine, vol. 359, no. 17, pp. 1811-1821, 2008.

[29] N. J. Pagidipati and E. D. Peterson, "Acute coronary syndromes in women and men," Nature Reviews Cardiology, vol. 13, no. 8, pp. 471-480, 2016.

[30] Y. Zhu, B. J. Pandya, and H. K. Choi, "Comorbidities of gout and hyperuricemia in the US general population: NHANES 2007-2008," The American Journal of Medicine, vol. 125, no. 7, pp. 679-687, 2012.

[31] R. Liu, C. Han, D. Wu et al., "Prevalence of hyperuricemia and gout in mainland China from 2000 to 2014: a systematic review and meta-analysis," BioMed Research International, vol. 2015, Article ID 762820, 12 pages, 2015.

[32] B. Liu, T. Wang, H. N. Zhao et al., "The prevalence of hyperuricemia in China: a meta-analysis," BMC Public Health, vol. 11, p. 832, 2011.

[33] A. G. Stack, A. Hanley, L. F. Casserly et al., "Independent and conjoint associations of gout and hyperuricaemia with total and cardiovascular mortality," QJM: An International Journal of Medicine, vol. 106, no. 7, pp. 647-658, 2013.

[34] J. Fang and M. H. Alderman, "Serum uric acid and cardiovascular mortality," JAMA, vol. 283, no. 18, pp. 2404-2410, 2000. 
[35] W. W. Au, D. Su, and J. Yuan, "Cigarette smoking in China: public health, science, and policy," Reviews on Environmental Health, vol. 27, no. 1, pp. 43-49, 2012.

[36] A. Norhammar, "Diabetes and cardiovascular mortality: the impact of sex," The Lancet Diabetes \& Endocrinology, vol. 6, no. 7, pp. 517-519, 2018.

[37] L. Tamariz, F. Hernandez, A. Bush, A. Palacio, and J. M. Hare, "Association between serum uric acid and atrial fibrillation: a systematic review and meta-analysis," Heart Rhythm, vol. 11, no. 7, pp. 1102-1108, 2014.

[38] A. J. Manolis, "Serum uric acid and atrial fibrillation," Current Medical Research and Opinion, vol. 33, no. 3, pp. 21-25, 2017.

[39] G. Huang, R. H. Xu, J. B. Xu et al., "Hyperuricemia is associated with atrial fibrillation prevalence in very elderly-a community based study in Chengdu, China," Scientific Reports, vol. 8, no. 1, p. 12403, 2018.

[40] L. Tamariz, S. Agarwal, E. Z. Soliman et al., "Association of serum uric acid with incident atrial fibrillation (from the Atherosclerosis Risk in Communities [ARIC] study)," The American Journal of Cardiology, vol. 108, no. 9, pp. 1272-1276, 2011.

[41] G. Giannopoulos, C. Angelidis, and S. Deftereos, "Gout and arrhythmias: in search for causation beyond association," Trends in Cardiovascular Medicine, vol. 29, no. 1, pp. 41-47, 2019.

[42] N. Li and D. Dobrev, "Hyperuricemia: a causal player or a bystander linking inflammatory signaling and atrial fibrillation?" International Journal of Cardiology, vol. 231, pp. 177-178, 2017.

[43] J.-Y. Youn, J. Zhang, Y. Zhang et al., "Oxidative stress in atrial fibrillation: an emerging role of NADPH oxidase," Journal of Molecular and Cellular Cardiology, vol. 62, pp. 72-79, 2013.

[44] W. Baldwin, S. McRae, G. Marek et al., "Hyperuricemia as a mediator of the proinflammatory endocrine imbalance in the adipose tissue in a murine model of the metabolic syndrome," Diabetes, vol. 60, no. 4, pp. 1258-1269, 2011.

[45] P. Korantzopoulos, K. P. Letsas, and T. Liu, "Xanthine oxidase and uric acid in atrial fibrillation," Frontiers in Physiology, vol. 3, p. 150, 2012.

[46] S. Yamada, H. Suzuki, M. Kamioka, Y. Kamiyama, S.-I. Saitoh, and Y. Takeishi, "Uric acid increases the incidence of ventricular arrhythmia in patients with left ventricular hypertrophy," Fukushima Journal of Medical Science, vol. 58, no. 2, pp. 101-106, 2012. 